\title{
Proficiency modulates between- but not within-language structural priming
}

\author{
Saoradh Favier (iD) A Aileen Wright $\cdot$ Antje Meyer $\cdot$ Falk Huettig
}

Received: 14 December 2018/Revised: 5 July 2019/Accepted: 24 July 2019/Published online: 5 August 2019

(C) The Author(s) 2019

\begin{abstract}
The oldest of the Celtic language family, Irish differs considerably from English, notably with respect to word order and case marking. In spite of differences in surface constituent structure, less restricted accounts of bilingual shared syntax predict that processing datives and passives in Irish should prime the production of their English equivalents. Furthermore, this cross-linguistic influence should be sensitive to L2 proficiency, if shared structural representations are assumed to develop over time. In Experiment 1, we investigated cross-linguistic structural priming from Irish to English in 47 bilingual adolescents who are educated through Irish. Testing took place in a classroom setting, using written primes and written sentence generation. We found that priming for prepositional-object (PO) datives was predicted by self-rated Irish (L2) proficiency, in line with previous studies. In Experiment 2, we presented translations of the materials to an English-educated control group $(\mathrm{n}=54)$. We found a within-language priming effect for PO datives, which was not modulated by English (L1) proficiency. Our findings are
\end{abstract}

S. Favier $(\bowtie) \cdot$ A. Meyer $\cdot$ F. Huettig

Max Planck Institute for Psycholinguistics, Nijmegen,

The Netherlands

e-mail: Saoradh.Favier@mpi.nl

\section{A. Wright}

School of Allied Health, University of Limerick,

Limerick, Ireland compatible with current theories of bilingual language processing and L2 syntactic acquisition.

Keywords Cross-linguistic structural priming Bilingualism · Proficiency $\cdot$ Shared syntax

\section{Introduction}

Structural priming and, perhaps most intriguingly, cross-linguistic structural priming, can be used as tools to investigate how the mind represents abstract syntactic information. While theories differ as to the underlying mechanisms of the effect, the persistence of syntactic structures within and between languages is well attested in the literature. To cite a classic example, Bock (1986) found that after hearing and repeating a sentence like The corrupt inspector offered a deal to the bar owner, participants were more likely to use a prepositional-object dative to describe an unrelated pictured event (e.g., The boy is handing a valentine to the girl), compared with its alternative, the double-object dative (The boy is handing the girl a valentine).

Since it was first reported over 30 years ago, the effect of recent syntactic experience on subsequent production has been demonstrated with a variety of tasks, syntactic structures, and languages (see Mahowald et al. 2016, for a meta-analysis). These effects, 
which occur in the absence of lexical or semantic repetition (Bock 1989; Bock and Loebell 1990), are taken as evidence for the representation of abstract structure in the language processing system (Branigan and Pickering 2017). Structural priming from one language to another suggests a further level of abstraction, at which some syntactic information is shared across languages.

Theoretical accounts of shared syntax in the bilingual mind are supported to varying degrees by the cross-linguistic syntactic priming literature (see Van Gompel and Arai 2018, for a review). One issue still subject to debate is the importance of syntactic congruency between languages, with implications for the scope of shared syntax in bilingualism. While some studies have found that the cross-linguistic priming effect depends on both languages sharing the same surface constituent structure (e.g., Loebell and Bock 2003; Bernolet et al. 2007), others have demonstrated structural priming between languages with major typological differences such as Korean and English (Shin and Christianson 2009), and Scottish Gaelic and English (Kutasi et al. 2018).

\section{Language proficiency}

A recent empirical and theoretical focus concerns the role of L2 proficiency in cross-linguistic structural priming. Hartsuiker and Bernolet (2017) hypothesised that L2 syntactic acquisition is characterised by the development of abstract structural representations that progressively become less language specific and more integrated with existing representations in L1. The presumed result is that similar structures in L1 and L2 share representations, which are activated during syntactic coding in either language, and thus facilitate cross-linguistic structural priming. It follows from this account that the magnitude of the priming effect should be modulated by L2 proficiency, if higher proficiency is associated with more abstract, languageindependent representations for the target structure.

An established index of proficiency is participants' self-rated language skills across modalities on a 7-point scale, which has been found to correlate with direct measures (Lemhöfer and Broersma 2012). There is some evidence to support the contribution of L2 proficiency to structural priming, from L1 to L2 and also within L2 (reviewed by Hartsuiker and Bernolet 2017). However, this has not been a consistent finding in the literature to date (e.g., Hartsuiker et al. 2016; Kutasi et al. 2018). We consider these studies in more detail in the discussion section.

Less studied populations in psycholinguistic research

The importance of gathering data from less studied populations is increasingly acknowledged in the cognitive sciences. In the domain of language processing, broadening the relevance of research on a global level requires an active focus on minority cultures and language communities. Multilingualism is the norm rather than the exception across most of the world, and speakers of minority languages account for a large part of this phenomenon (e.g., in India; Pandharipande 2002). These communities by their nature are small, sometimes difficult to access, and may require alternatives to traditional lab-based testing. Regardless of the challenges, data from previously unstudied groups is essential to develop theories of language processing that take into account the diversity of human language and cognitive abilities.

In a literature largely dominated by majority languages, it is not surprising that speakers of Irish Gaelic (henceforth, Irish) have not been a focus of any language processing research to date. Yet, the changing demographic distribution of the speaker community, as well as the typological distance between Irish and English, make this an interesting case study in bilingualism.

Irish is the national language of the Republic of Ireland and a recognised minority language in Northern Ireland. It is the oldest of three Goidelic languages (the others being Scottish Gaelic and Manx), which belong to the Celtic branch of Indo-European. Notable typological differences from English include verb-subject-object (VSO) word order and the use of case marking.

Despite its official status as the first language of Ireland, Irish is more widely spoken as an L2, with proficiency and frequency of use varying greatly across speakers. In a survey published by the European Commission, $22 \%$ of respondents in Ireland reported some ability to speak Irish, while only $3 \%$ described it as their first language (Eurobarometer 2012). L1 Irishspeaking communities exist predominantly in western coastal regions of Ireland, collectively known as the Gaeltacht. While the numbers of L1 speakers in these rural communities is in rapid decline, the growing 
popularity of Irish-medium education outside of the Gaeltacht has produced a generation of 'new speakers' of Irish, concentrated in urban areas (Slatinská 2017).

In this paper, we investigate cross-linguistic structural priming in a sample of 'new speakers' of Irish: bilingual adolescents attending an urban, Irish-medium secondary school. Adolescents arguably constitute another under-represented group in the language processing literature, often falling outside the remit of both developmental and adult studies. We cannot assume that findings from the adult literature would necessarily generalise to adolescent language processing, especially given the common practice of sampling university undergraduates, who represent a relatively restricted range of language experience and ability. Adolescent performance on language processing tasks is likely to be subject to more variability than is seen in the typical, highly educated young adult sample.

\section{The current study}

This paper focuses on two well-studied structures in the cross-linguistic syntactic priming literature: datives and passives. Dative priming usually relies on the structural alternation whereby the same ditransititve event can be described using either a double-object (DO) or prepositional-object (PO) construction (e.g., The monk gave the cowboy a cake vs. The monk gave a cake to the cowboy). In contrast to English, Irish permits only one type of dative (1), which corresponds most closely to the English PO construction in terms of constituent order (Direct Object + Indirect Object). However, surface constituent structure diverges from the English PO, since Irish uses case marking, rather than a preposition, to specify the indirect object (the dative-inflected article don, which has no correspondence in English). Furthermore, as per Irish VSO word order, the main verb (Thug, "gave" in 1) occurs sentence-initially, marking a salient difference from the English translation equivalent.

1. Thug an manach cáca don buachaill bó.

Gave the monk cake to the cowboy

"The monk gave a cake to the cowboy"
The Irish passive (2) shares some structural features with its English equivalent. Aside from the initial position of the verbal auxiliary Bhí ("was"), the Irish passive construction has a similar constituent order to English: patient in the subject position, followed by a participle (buailte, "hit" in 2), and a prepositional "by" phrase that specifies the agent (ag corresponds to "by"). As Irish passives are very rarely used in the present tense, we focus on the past tense in this study.

2. Bhí an fear grinn buailte ag an mairnéalach.

Was the clown hit by the sailor

"The clown was hit by the sailor"

Using a comprehension-to-production priming paradigm, we examined the extent to which reading dative and passive sentences in Irish influenced students' subsequent syntactic choices in English, in a written sentence generation task (Experiment 1). As demonstrated above, Irish datives and passives overlap with their English counterparts to varying degrees, but it is clear that surface structure is not identical across languages. We based our predictions on previous evidence for cross-linguistic priming in the absence of shared surface structure (Kutasi et al. 2018; Shin and Christianson 2009, but see Bernolet et al. 2007). Firstly, we predicted that Irish dative sentences would prime the production of PO datives in English. Specifically, we expected to see an increase in the proportion of PO responses following an Irish dative prime, compared to a structurally unrelated baseline condition (comprising three conjoined noun phrases). Secondly, on active-passive trials, we predicted an effect of prime type on the structure of responses, such that more English passive sentences would be produced following an Irish passive prime than an active or baseline prime (two conjoined noun phrases). As the strongly preferred canonical form, we did not expect to observe a priming effect for actives (production too near ceiling). We included a measure of self-rated Irish proficiency in order to test the prediction that cross-linguistic structural priming is modulated by L2 proficiency.

Experiment 2 is a within-language control experiment, which we conducted with Irish adolescents receiving their education through English. Experiment 
2 used the same design and procedure as Experiment 1, but we presented prime sentences in English instead of Irish. This between-experiment comparison allowed us to examine the specific contribution of the cross-linguistic design to our results in Experiment 1. We were also interested in the contribution of L1 proficiency to within-L1 priming. Due to anticipated ceiling effects on the self-rated proficiency measure, we used participants' most recent standardised English exam grades as a proxy for L1 proficiency in Experiment 2.

The Junior Certificate is the standardised assessment of academic attainment in Ireland, which participants had completed between 6 and 18 months prior to the current study. Although the Junior Certificate English curriculum also encompasses literature and media studies, four of the seven components of the exam directly assess reading comprehension and functional writing. Moreover, quality of written language is applied as a marking criterion to all components. Aspects of linguistic competence highlighted in the marking scheme include syntactic complexity, discourse structure, coherence, and spelling. We therefore considered Junior Certificate English grades as a reasonable proxy for L1 proficiency, providing an overall picture of participants' language aptitude, despite the potential noise introduced by less relevant components of the assessment such as literature. These grades were included as an exploratory covariate in the priming analyses in Experiment 2.

\section{Experiment 1}

We investigated structural priming from Irish to English, using written sentence generation in a classroom setting. Ethical approval for the study was granted by the Education and Health Sciences Research Ethics Committee at the University of Limerick, Ireland.

Methods

\section{Participants}

Forty-seven English-Irish bilinguals (27 females) gave informed consent to participate in the study. The participants, aged $16-17$ years $(\mathrm{M}=16.37$,
$\mathrm{SD}=0.49$ ), were recruited from an Irish-medium secondary school in Dublin, Ireland. Written consent was obtained both from participants and their parents/guardians. We used a questionnaire (adapted from Kutasi et al. 2018; “Appendix 2") to assess Irish language history, frequency of use, and selfrated proficiency across speaking, listening, reading, and writing on a 7-point scale. Table 1 summarises the results of the questionnaire, which was completed by thirty-six participants. The overall mean for self-rated Irish proficiency across modalities was $5.98(\mathrm{SD}=0.70)$. As they received their education through Irish, all participants engaged in Irish conversation daily, and twenty participants also reported speaking Irish at home. The majority were sequential bilinguals, who began acquiring Irish at school, while three had acquired Irish and English simultaneously from birth. Participants were also asked to provide their Junior Certificate exam results for Irish and English. Of those who provided their Junior Certificate exam grades, all had achieved either a B $(23 \%)$ or a C $(77 \%)$ in English.

\section{Design and materials}

There were two sets of experimental materials, targeting active/passive priming and PO dative respectively. The items of the two sets were mixed, together with a set of filler items. Each item consisted of a prime picture, a prime sentence, and a target picture with a written verb printed above it. These elements are described in more detail in the following sections. Note that prime and target pictures and target verbs were displayed on a screen to the entire group of participants simultaneously. Written prime sentences were presented in participants' answer booklets. Their cover task was to provide a true/false response to each sentence, based on congruity with the displayed picture. The answer booklet contained empty spaces for participants' written descriptions of target pictures.

Active-passive set We created 48 transitive items for the active-passive set, which consisted of prime sentences in Irish and their corresponding pictures, paired with target pictures to elicit sentences in English. Picture stimuli were black and white line drawings of transitive events involving human agents and patients (adapted from Bernolet et al. 2016, and Kutasi et al. 2018). As in Kutasi et al. patients always 
Table 1 Profile and self-rated Irish language proficiency of participants in Experiment $1(n=36)$

\begin{tabular}{lll}
\hline Variable & Mean (SD) & Range \\
\hline Age at testing & 16.37 & $16-17$ \\
& $(0.49)$ & \\
Age when began acquiring Irish (years) & $3.45(1.22)$ & $0-5$ \\
Speaking proficiency (7pt) & $6.03(0.86)$ & $4-7$ \\
Listening proficiency (7pt) & $6.42(0.61)$ & $5-7$ \\
Reading proficiency (7pt) & $6.03(0.98)$ & $4-7$ \\
Writing proficiency (7pt) & $5.51(1.12)$ & $3-7$ \\
\hline
\end{tabular}

appeared on the left, to increase the likelihood of eliciting passives.

There were three versions of each prime sentence in Irish, corresponding to the three experimental conditions: Active, Passive, and Baseline (see Fig. 1). Prime sentences in the Active condition described pictured events using the canonical order (verb + agent + patient). Prime sentences in the Passive condition described the same events using a passive construction with the agent in a prepositional phrase (see Example 2 in the Introduction). To provide a neutral control condition without any verb, Baseline sentences were noun phrase conjunctions referring to the two characters depicted, e.g., Gadaí agus póilín, “A burglar and a policeman"). The characters were named in the same order that they appeared in the pictures, from left to right. Noun phrases used the indefinite article (unmarked in Irish) for greater naturalness.

Prime pictures depicted six actions: punch, chase, kiss, push, tickle, and pull. We selected a further six actions for the target pictures, to elicit English sentences: carry, weigh, clean, kick, follow, and hit. The respective target verb was printed in English above each target picture. Across the item set, comprising 48 prime pictures and 48 target pictures, there were eight different depictions of every action.

The order of prime pictures was pseudo-randomised such that the same action was depicted no more than twice consecutively. To each prime picture we then assigned a different target picture. The final item set contained most possible combinations of prime and target actions, except for pairs with too close a semantic relationship (e.g., chase-follow; punch-hit), which we excluded.

A set of 20 human characters (e.g., sailor, dancer, monk) appeared as the agents and patients of both prime and target actions. Agents and patients were repeated across items, with the constraint that a character could appear no more than twice in the same role for each target action. Within items, there
Fig. 1 Example item from the active-passive set: Prime picture (top), corresponding Irish prime sentences, and target picture (bottom) with printed verb to elicit an English sentence

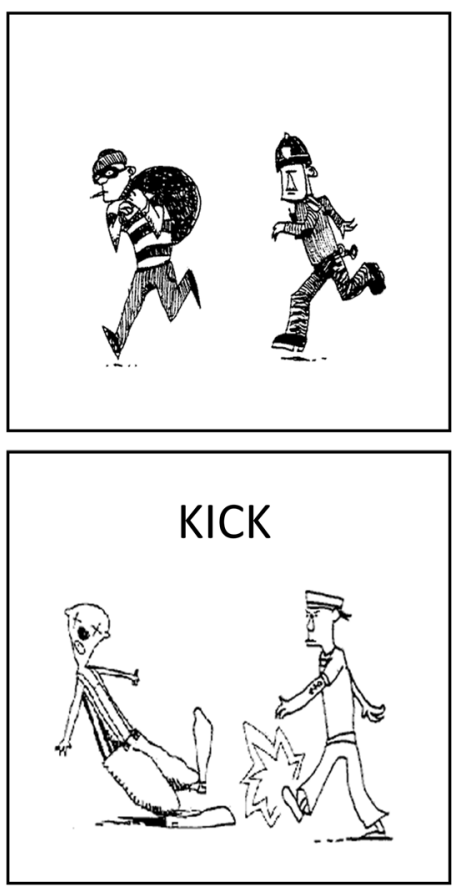

\section{1a. Active}

Lean an póilín an gadaí
Chased the policeman the burglar
"The policeman chased the burglar"

\section{1b. Passive}

Bhí an gadaí leanta ag an bpóilín

Was the burglar chased by the policeman

"The burglar was chased by the policeman"

1c. Baseline

Gadaí agus póilín.

A burglar and a policeman 
was no repetition of action or characters between prime and target pictures.

Dative set We created 48 dative items with the same structure as active-passive items (prime sentences in Irish, prime pictures, and target pictures). Picture stimuli were black and white line drawings of ditransitive events, adapted from Bernolet et al. (2016). They involved a human agent, an object theme, and a human recipient. In dative pictures, the agent always appeared on the left, the theme in the middle, and the recipient on the right.

There were two versions of each prime sentence, corresponding to two experimental conditions: Dative and Baseline (see Fig. 2). Sentences in the Dative condition took the Irish canonical form (verb + agent + theme + recipient $)$, which corresponds most closely to the prepositional-object dative in English. As in the active-passive set, the Dative Baseline condition used conjoined noun phrases without a verb. However, these consisted of three noun phrases rather than two, to match the number of entities named in the Dative condition (Baseline naming order: agent, recipient, and theme).

Prime pictures depicted six actions, corresponding to dative verbs that alternate in English: give, sell, show, pass, throw, and offer. We selected a further six alternating dative verbs as targets, and superimposed them on the target pictures: hand, lend, award, grant, owe, and allocate. There were 48 prime pictures and 48 target pictures, so every action appeared eight times across the item set.

We avoided target verbs with a strong bias towards either the English prepositional-object (PO) or doubleobject (DO) dative, using results of a corpus analysis by Gries and Stefanowitsch (2004) to inform our selection. The overall rate of $\mathrm{PO}$ occurrences across all alternating dative verbs in the one-million-word ICEGB corpus was $65 \%$. Table 2 shows the percentage of PO occurrences reported in that study for our selected prime and target verbs. Our target verbs ranked amongst the least biased of all the alternating dative verbs analysed by Gries and Stefanowitsch. We also conducted a Google Books search to ensure that the transitional probabilities of DO and PO constructions were similar for each item, i.e. the probability of a target verb occurring adjacently to a given recipient versus a given theme.
The order of dative prime pictures was pseudorandomised, as in the active-passive set, and each prime picture was paired with a target picture. As dative verbs inherently share semantic features, it was impossible to avoid some overlap in meaning within prime-target pairs. Prime and target actions therefore occurred in all combinations at least once across the dative set.

The human characters described above were used as agents and recipients in the dative set. These were repeated across items, but did not perform the same role more than twice in any given action. Six object themes (cup, apple, jug, book, banana, hat) were also repeated across items, but never appeared more than twice with the same verb. Within items, there was no repetition of actions or entities between prime and target pictures.

Fillers We used 48 additional filler items, comprising 'prime' pictures of two or more noninteracting entities, 'prime' noun phrases in Irish (e.g., beirt mhanaigh, "two monks"), and 'target' pictures of single entities. For consistency with the critical items, 'target' pictures in the filler set also appeared with a printed English verb. Fillers used only intransitive verbs, unlikely to elicit transitive or dative descriptions (e.g., walk). To facilitate the 'True or False?' cover task, all filler items contained mismatching 'prime' pictures and sentences. We shuffled picture/sentence pairs so that one or more of the entities depicted mismatched the written description.

List construction The active-passive, dative, and filler sets were mixed to create a master list containing 144 items (288 pictures in total). The list was constructed such that no two transitive or dative items occurred consecutively.

From the master list we derived six experimental lists using a $3 \times 2$ Latin square design, so that across the lists every transitive item occurred twice in each of the three experimental conditions (Active, Passive, and Baseline), and every Dative item occurred three times in each of the two experimental conditions (Dative and Baseline). The lists were pseudo-randomised such that all experimental conditions were evenly distributed within each list. 
Fig. 2 Example item from the dative set: prime picture (top), corresponding Irish prime sentences, and target picture (bottom) with printed verb to elicit an English sentence
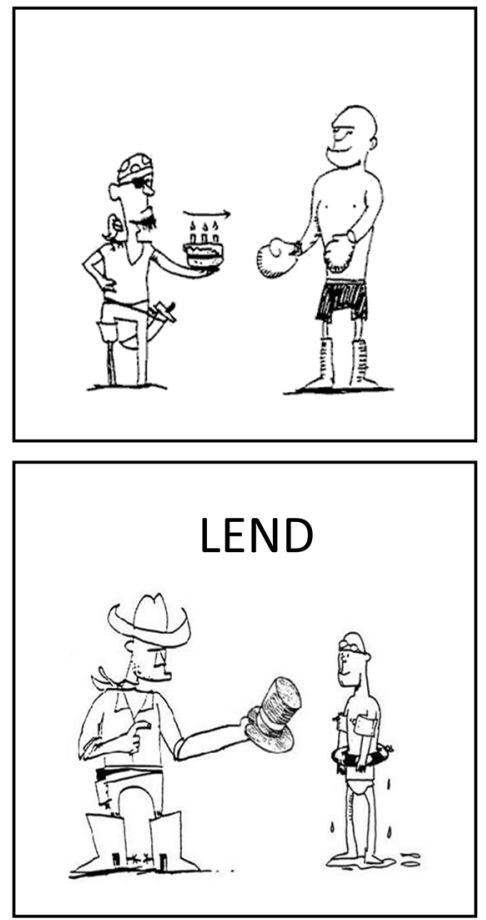

\section{2a. Dative}

Thug an foghlaí mara cáca don dornálai Gave the pirate cake to the boxer

"The pirate gave a cake to the boxer"

\section{2b. Baseline}

Dornálaí, an foghlaí mara, agus cáca A boxer, a pirate and a cake
Table 2 Percentage of PO occurrences for each prime and target verb, based on the one-million-word ICE-GB corpus

\begin{tabular}{llll}
\hline Prime verb & $\%$ PO & Target verb & $\%$ PO \\
\hline Give & 24.1 & Award & 30.0 \\
Show & 23.4 & Grant & 28.6 \\
Offer & 25.9 & Allocate & 55.6 \\
Sell & 92.1 & Owe & 60.0 \\
Pass & 93.6 & Hand & 80.8 \\
Throw $^{\text {a }}$ & - & Lend & 65.0 \\
\hline
\end{tabular}

The overall rate of $\mathrm{PO}$ occurences across all alternating dative verbs in the corpus was $65 \%$ (from Gries and Stefanowitsch 2004, p 106, Table 2)

${ }^{\mathrm{a}}$ Gries and Stefanowitsch do not provide data on throw; however, it is attested as an alternating dative verb in other corpus analyses (e.g., Lapata 1999)

Trial structure We used a similar paradigm to previous comprehension-to-production priming studies (e.g., Bernolet et al. 2016), whereby participants alternately comprehend and produce picture descriptions. At the start of a trial, participants in the present study saw a prime picture displayed for $7 \mathrm{~s}$, and read the corresponding Irish prime sentence, to which they responded 'true' or 'false'. Next, a target picture was presented for $13 \mathrm{~s}$, and participants wrote a sentence in English to describe the picture, making use of the verb provided. The experiment consisted of 144 trials, preceded by six practice trials, which followed the same two-part structure. Figures 3 and 4 describe the composition and timing of example transitive and dative trials respectively.

Presentation of materials We created the alternating list of prime and target pictures in Microsoft PowerPoint, with target verbs printed in capital letters above the target pictures (Calibri, 60 point font). The pictures were numbered in order from 1 to 288. The number was displayed in a box in the top left hand corner of the picture (Calibri bold, 44 point font). We set timings such that each prime picture was displayed for $7 \mathrm{~s}$, immediately followed by a target picture, displayed for $13 \mathrm{~s}$. The file was converted to a movie format, playable through QuickTime Player. We projected the movie onto a large screen at the front of the classroom, visible to all participants.

All prime and target pictures were presented to all participants in a fixed order, to facilitate group participation. Written prime sentences were presented to each participant individually in an answer booklet. Whereas the order of items was the same for all participants, they read different versions of the prime 
Fig. 3 Example transitive trial structure (passive prime condition)
Fig. 4 Example dative trial structure (dative prime condition)

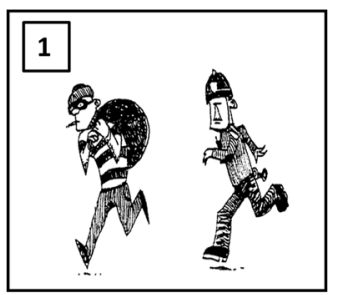

- Prime picture presented on screen.

- Participant reads corresponding Irish prime sentence in answer booklet (translation: 'The burglar was chased by the policeman').

- Participant indicates whether the prime sentence correctly describes the prime picture by ticking 'true' or 'false'.

1. Bhí an gadaí leanta ag an bpóilín.

$\begin{array}{ll}\text { true } & \text { false } \\ \checkmark & \square\end{array}$

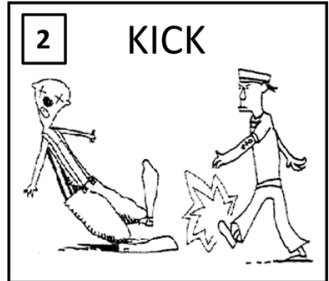

- After 7 seconds, the target picture appears on the screen.

- In the corresponding section of the answer booklet, the participant writes an English sentence to describe the target picture, using the printed verb (example response below).

- After 13 seconds, the next trial begins.

2. Describe in English.

The clown was kicked by the sailor.

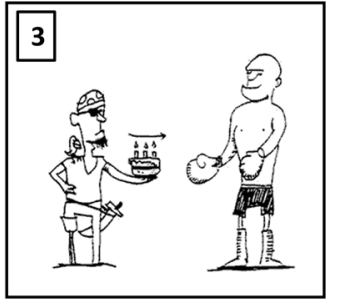

- Prime picture presented on screen.

- Participant reads corresponding Irish prime sentence in answer booklet (translation: 'The pirate gave a cake to the boxer').

- Participant indicates whether the prime sentence correctly describes the prime picture by ticking 'true' or 'false'.

\begin{tabular}{|lll|}
\hline 3. Thug an foghlaí mara cáca don dornálaí. & true & false \\
\hline
\end{tabular}

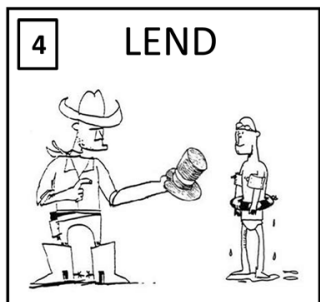

- After 7 seconds, the target picture appears on screen.

- In the corresponding section of the answer booklet, the participant writes an English sentence to describe the target picture, using the printed verb (example response below).

- After 13 seconds, the next trial begins.
7 seconds

13 seconds

7 seconds

13 seconds

The cowboy lent a hat to the swimmer. 
sentence for a given item. The versions of the prime sentences presented in each answer booklet corresponded to one of the six experimental lists. There were therefore six unique answer booklets, each containing 144 prime sentences, two per page. The six versions of the booklet were randomly but equally distributed, so that eight participants completed each version.

Two boxes, labeled 'true' and 'false', appeared next to each prime sentence in the answer booklet, to facilitate the picture verification cover task. Underneath each prime sentence was the printed instruction Describe in English, and empty space for participants to write down their description of the corresponding target picture (see "Appendix 1" for page layout). The alternating prime sentences and blank spaces in the answer booklet were numbered from 1 to 288 , corresponding to the numbers displayed in the top left hand corner of prime and target pictures. This was intended to ensure that participants responded to each picture in the appropriate place in their answer booklets. The answer booklets were printed in black and white on A4 paper and all text was in 12 point, Calibri font.

\section{Procedure}

All participants were tested simultaneously in a large classroom in their school, seated at desks facing the front of the room. They attended to verbal instructions and completed the experimental tasks independently and in silence. The experimenter ran the session, with additional supervision provided by three class teachers.

Every participant received a pen and an answer booklet, containing only the written prime sentences, and empty spaces for responses to the prime task and the target task. All pictures were projected onto a large screen at the front of the classroom, with pictures and text visible to participants seated at the back of the room. Both oral and written instructions were provided at the start of the experiment, followed by six practice trials to familiarise participants with the task and characters. The practice block comprised one dative trial, one transitive trial with an active prime, and one with a passive prime. These were interleaved with three filler trials. After the practice block, participants had the opportunity to ask questions about the task.
After the experimental task (approximately $50 \mathrm{~min}$ ), there was a 10-minute break. Participants then completed the Irish Language History Questionnaire (see "Appendix 2"). The whole session lasted $1 \mathrm{~h}$ and $30 \mathrm{~min}$.

Results and discussion

Overall, participants performed well on the true/false cover task, in which they judged whether an Irish sentence correctly described a given picture. The mean accuracy score was $87.69 \%(\mathrm{SD}=16.62 \%)$ on active-passive trials and $92.34 \%(\mathrm{SD}=7.08 \%)$ on dative trials. Within the active-passive set, accuracy was lowest on passive trials $(86.5 \%)$, while on active trials it was comparable to performance on the dative set $(92.94 \%)$. This difference could reflect an increased processing load for passive sentences, associated with their low frequency in Irish. Nevertheless, the generally high accuracy rate suggests that participants understood the prime sentences in most cases. In the following analyses we included all trials with scorable responses, regardless of accuracy on the true/false task, since excluding incorrect trials did not change the pattern of results.

Transitive event descriptions were scored as active if they featured the agent in subject position, followed by the verb, and the patient in object position (e.g., The dancer pushed the waitress). Descriptions were scored as passive if they featured the patient as the subject of the sentence, the verb, and the agent in a prepositional by phrase (e.g., The teacher was weighed by the nun). Non-transitive descriptions were scored as 'other', as were descriptions that only referred to one entity in the picture. This included patient-focusing constructions that omitted the agent (i.e. short passives), in accordance with the scoring criteria typically applied in adult priming studies (e.g., Hartsuiker et al. 2004). We also carried out analyses based on a more lenient scoring scheme, often used in the developmental priming literature (e.g. Branigan and McLean 2016). Under lenient scoring, short passives that omitted the by-phrase (e.g., The boxer got hit) were counted along with full passives.

Participants produced 1909 descriptions, of which 1516 were active $(79.41 \%), 46$ were full passives (2.41\%), 114 were short passives (5.97\%), and 233 were scored as 'other' responses under the lenient scheme (18.17\%). Table 3 displays the frequency and 
proportion of passive, active, and 'other' responses following each prime type, based on strict and lenient scoring schemes separately. The rate of passive production was very low across all conditions, although marginally higher after a passive prime than after an active or baseline prime.

Due to the very small number of full passives produced in Experiment 1, there were insufficient data points to conduct a reliable analysis of structural priming based on the strict scoring scheme. We carried out an exploratory analysis of priming under the lenient scoring scheme by fitting a mixed logit model to the re-coded response data; however, we did not find a main effect of passive priming nor an interaction between priming and proficiency. It is conceivable that this result reflects the small number of observations per condition, even with the inclusion of short passives, resulting in insufficient power.

Dative event descriptions were scored as double object (DO) if they contained the agent, the verb, and the recipient immediately followed by the theme (e.g., The cook lends the boxer a cake). Descriptions were scored as prepositional object (PO) when the theme was named first, followed by the recipient in a prepositional to phrase (e.g., The painter handed a jug to the dancer). Non-dative descriptions, and those that did not name all three entities, were scored as 'other'.

Participants produced 1718 descriptions, of which 751 were scored as double-object datives (43.71\%), 345 as prepositional-object datives (20.08\%), and 622 as 'other' responses (36.2\%). Table 4 shows the frequency and proportion of PO, DO, and 'other' responses following baseline and dative primes.

The relatively high proportion of 'other' responses in the dative set is partly due to the frequency of incomplete responses, missing at least one argument (23.28\%). It is possible that some participants did not manage to write down a complete dative sentence containing three entities within the time allowed. We also observed legitimate descriptions of dative events involving passive constructions (160 instances, e.g., The painter was awarded a banana). This may reflect a tendency of specific target verbs to attract the passive, or the transfer of a passive priming effect to subsequent dative trials. However, evidence for the rapid decay of priming in written production (Branigan et al. 1999) makes this unlikely to be a "leaked" effect of previous exposure to passive sentences, as exploratory analysis revealed that passive descriptions of dative events were not any more prevalent when the preceding trial was passive than when it was active.

\section{Mixed logit model of dative responses}

We fit a generalised logistic mixed model to predict the occurrence of PO responses in the dative dataset, using the 'Ime4' package in R, version 1.0.153 (Bates et al. 2013). PO responses were as coded as ' 1 ' and DO and all other responses were coded as ' 0 '. The model included random intercepts for items, target verbs, and participants, as well as by-target verb and by-participant random effects of Prime Type. Prime Type (Baseline/Dative) was a fixed factor in the model. We included self-rated Irish Proficiency as a continuous predictor, to investigate the relationship between L2 proficiency and cross-linguistic structural priming. Irish Proficiency was averaged across speaking, listening, reading, and writing, and entered into the model as a mean-centred score. 11 participants were excluded from the modelling analysis due to missing language history data.
Table 3 Frequency and proportion of passive, active, and 'other' responses by prime condition, based on strict and lenient scoring schemes

\begin{tabular}{|c|c|c|c|c|c|c|}
\hline & \multicolumn{2}{|l|}{ Baseline } & \multicolumn{2}{|l|}{ Active } & \multicolumn{2}{|l|}{ Passive } \\
\hline & Frequency & Proportion & Frequency & Proportion & Frequency & Proportion \\
\hline \multicolumn{7}{|l|}{ Strict scoring } \\
\hline Passive response & 14 & 0.02 & 12 & 0.02 & 20 & 0.03 \\
\hline Active response & 509 & 0.80 & 519 & 0.81 & 488 & 0.77 \\
\hline Other response & 116 & 0.18 & 108 & 0.17 & 123 & 0.19 \\
\hline \multicolumn{7}{|l|}{ Lenient scoring } \\
\hline Passive response & 51 & 0.07 & 47 & 0.06 & 62 & 0.08 \\
\hline Active response & 509 & 0.80 & 519 & 0.81 & 488 & 0.77 \\
\hline Other response & 79 & 0.13 & 73 & 0.13 & 81 & 0.15 \\
\hline
\end{tabular}


Table 4 Frequency and proportion of prepositional-object (PO), double-object (DO), and 'other' responses by prime condition

\begin{tabular}{llllll}
\hline & \multicolumn{2}{l}{ Baseline } & & & Dative \\
\cline { 2 - 3 } \cline { 5 - 5 } & Frequency & Proportion & & Frequency & Proportion \\
\hline $\begin{array}{l}\text { response } \\
\text { DO } \\
\text { response }\end{array}$ & 155 & 0.18 & 190 & 0.22 \\
$\begin{array}{c}\text { Other } \\
\text { response }\end{array}$ & 323 & 0.38 & 299 & 0.35 \\
\hline
\end{tabular}

The model results are summarized in Table 5. The negative intercept reflects a baseline preference for the DO dative. There is a small positive effect of Prime Type on the log-odds likelihood of a PO response, based on the $95 \%$ confidence interval for the coefficient having a lower bound very near to zero. While self-rated Irish proficiency does not predict the use of PO datives in English, the model results indicate a positive interaction between Irish proficiency and Prime Type. That is, participants with higher self-rated proficiency were more likely to produce an English PO dative after reading an Irish dative prime, than after a non-dative baseline prime. This result suggests that the observed $4 \%$ difference in PO production between conditions (shown in Table 4) was largely driven by the higher-proficiency participants. Figure 5 illustrates the positive relationship between self-rated Irish proficiency and individual differences in the magnitude of the PO priming effect.

In sum, our results indicated a small effect of dative priming, which was modulated by self-rated proficiency in Irish, the priming language. Despite its considerably different surface structure, the Irish dative may be more connected to the English PO than to the DO dative in abstract representational space, as a result of congruent constituent order (Direct object + Indirect Object). Interpreting our results within the developmental model of L2 syntactic acquisition (Hartsuiker and Bernolet 2017), this structural overlap had a more facilitating effect for higher-proficiency participants because they are equipped with more abstract representations of Irish (L2) structures, presumably allowing for a greater degree of connectedness with existing representations of English (L1) structures.

We did not find the same pattern of results in the active-passive set. Overall, the rate of passive production was very low. Even under a more lenient scoring system that included short passives, there was only a marginal difference in the proportion of passive responses following a passive prime relative to baseline. In addition, exploratory analysis did not show evidence for an interaction with Irish proficiency, as we observed for dative priming. However, we interpret this result with caution given the number of data points, which was arguably insufficient to assess interaction effects reliably.

It is not clear whether the weak evidence for crosslinguistic priming in Experiment 1 reflects the linguistic distance between Irish and English or methodological factors, such as the use of written sentence generation in a classroom setting. To address this question, we conducted a within-language control experiment (Experiment 2). We used the same design and procedure as Experiment 1, with English-only materials and an age-matched group of students attending English-medium schools. If we find much stronger evidence for priming within language than between languages, we might conclude that the representations of equivalent structures in English and Irish are connected but not fully integrated, perhaps due to insufficient overlap in surface structure,

Table 5 Summary of fixed effects in the mixed logit model $(\mathrm{N}=1293$, log-likelihood $=-555.9)$

\begin{tabular}{lcccc}
\hline Predictor & Coefficient & SE & $z$ value & $95 \%$ CI \\
\hline Intercept & -2.12 & 0.46 & -4.61 & -3.02 to -1.22 \\
Prime type: dative & 0.36 & 0.20 & 1.78 & -0.04 to 0.76 \\
Irish proficiency & -0.62 & 0.38 & -1.60 & -1.37 to 0.14 \\
Prime type: dative $\times$ Irish proficiency & 0.53 & 0.23 & 2.32 & 0.08 to 0.98 \\
\hline
\end{tabular}

The intercept represents the log-odds of a PO response in the non-dative baseline condition (NP NP NP) for a participant with average Irish proficiency

$S E$ standard error, $C I$ confidence interval 


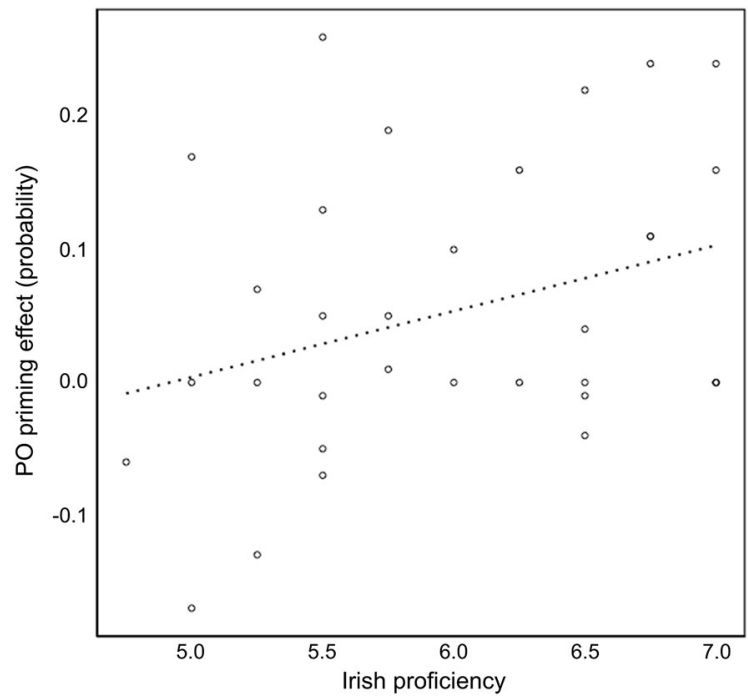

Fig. 5 Irish-to-English PO priming effect as a function of selfrated Irish proficiency (7-point scale, averaged across speaking, listening, reading, and writing). The priming effect is the probability of producing a $\mathrm{PO}$ dative in the dative priming condition, minus the baseline probability. Participants who produced a higher proportion of POs at baseline than after a dative prime thus show a negative effect

which might restrict cross-linguistic priming. If, on the other hand, we do not observe the expected main effect of within-language structural priming in Experiment 2 , the most likely conclusion will relate to methodological factors.

\section{Experiment 2}

Experiment 2 investigated within-language structural priming (English to English), using written sentence generation in a classroom setting.

Methods

\section{Participants}

54 native English speakers (32 female), aged 14-17 $(\mathrm{M}=16.02, \mathrm{SD}=0.76)$ were recruited from two English-medium secondary schools in the mid-west and south east of Ireland. Informed written consent was obtained from students and their parents prior to participation. As Irish is taught as a compulsory subject in most schools at primary and secondary level, all participants had had some degree of exposure to the language. However, based on responses to the Irish Language History Questionnaire (summarised in Table 6), participants' self-rated proficiency $(\mathrm{M}=4.27, \mathrm{SD}=1.53)$ was on average lower and more variable than that of the Irish-educated group in Experiment 1. We compared the mean proficiency ratings for the two groups using a Welch's $T$ test $(t=2.89)$. The standardised effect size (1.36) indicates that the magnitude of the difference is large. Furthermore, in contrast to the Irish-educated group's daily use of Irish, two-thirds of the English-educated group reported rarely using Irish in conversation, while the remaining third did so only in weekly Irish lessons at school. Therefore, we conclude that participants in Experiment 2 differ substantially from participants in Experiment 1 in terms of their selfrated Irish language proficiency, experience, and frequency of use. Standardised exam results for English suggest that academic performance was also more variable in this group, with grades ranging from A to E. Amongst the 49 participants who responded, the distribution of grades was A: $6 \%, \mathrm{~B}: 27 \%, \mathrm{C}: 39 \%$; D: $27 \%$; E: $2 \%$.

\section{Design and materials}

The design and materials were identical to those described in Experiment 1, except that the prime sentences had been translated into English.

\section{Procedure}

Participants were tested in their school classrooms, in two groups of 30 and 24 students, respectively. The set-up and procedure were the same as for Experiment 1, except that the cover task required participants to respond 'true' or 'false' to English descriptions of the prime pictures. As in Experiment 1, participants generated written descriptions to target pictures in English.

Results and discussion

Overall, accuracy on the true/false cover task was high, indicating that participants read and understood the prime sentences. The mean accuracy score was $96.21 \%$ ( $\mathrm{SD}=4.18 \%$ ) on active-passive trials and $96.10 \%(\mathrm{SD}=7.75 \%)$ on dative trials. $\mathrm{As}$ in 
Table 6 Profile and selfrated Irish language proficiency of participants in Experiment $2(n=50)$

\begin{tabular}{lcr}
\hline Variable & Mean (SD) & Range \\
\hline Age at testing & $16.02(0.76)$ & $14-17$ \\
Age when began acquiring Irish (years) & $4.88(1.29)$ & $3-10$ \\
Speaking proficiency (7pt) & $4.24(1.62)$ & $1-7$ \\
Listening proficiency (7pt) & $3.94(1.65)$ & $1-7$ \\
Reading proficiency (7pt) & $4.60(1.29)$ & $1-7$ \\
Writing proficiency (7pt) & $4.30(1.49)$ & $3-7$ \\
\hline
\end{tabular}

Experiment 1, the analyses included all trials with scorable responses, regardless of accuracy on the true/false task.

We used the same coding schemes described in Experiment 1 to score participants' written descriptions of transitive and ditransitive events. Participants produced 2457 transitive event descriptions, of which 1952 were active $(79.45 \%), 104$ were full passives (4.23\%), 155 were short passives $(6.31 \%)$, and 246 were scored as 'other' responses under the lenient scoring scheme $(10.00 \%)$. Under the strict scoring scheme, 'other' responses included short passives, as well as non-transitive and incomplete sentences. The frequency and proportion of passive, active, and 'other' responses following each prime type are displayed for both strict and lenient scoring schemes in Table 7. Across conditions, we observed very few full passives; however, there was a numerical trend towards producing more passives after passive primes than after active or baseline primes. As both scoring schemes yielded the same pattern of results, below we report analysis based on the strict scoring scheme.

\section{Mixed logit model of passive responses}

We used the 'Ime4' package in $\mathrm{R}$ to fit a generalised logistic mixed model to the active-passive dataset. Full passive responses (e.g., The boxer was hit by the cowboy) were as coded as ' 1 ', and active and all 'other' responses were coded as ' 0 '. The model included random intercepts for items, target verbs and participants, and a by-participant random effect of Prime Type. We added a random effect of Prime Type by School to account for the possible variance introduced by testing participants in two different schools. The fixed factor in the model was Prime Type (Baseline/Active/Passive), with Baseline taken as the reference level. We recoded participants' English exam grades (A-E) as a numeric score (5-1), This new variable, English Score, was mean-centred and entered into the model as a continuous covariate, including an interaction with Prime Type.

The model results summarised in Table 8 show that the log-odds of producing a full passive in the baseline condition was well below zero, reflecting the observed strong preference for actives. Since the $95 \%$ confidence interval for the Prime type: passive coefficient encompasses zero, we cannot conclude that there was a within-language passive priming effect. Thus, these results match those of Experiment 1 . In addition, the model results show no evidence for a main effect of English Score on the likelihood of a passive response, nor an interaction between English score and either Prime Type.

Participants produced 2351 ditransitive event descriptions, of which 719 were scored as doubleobject datives (30.58\%), 798 as prepositional-object datives (33.94\%), and 833 as 'other' responses $(35.43 \%)$. Table 9 displays the frequency and proportion of PO, DO, and 'other' responses following baseline and dative primes. The proportions indicate an $8 \%$ increase in the production of $\mathrm{PO}$ datives in the $\mathrm{PO}$ prime condition relative to the non-dative baseline.

\section{Mixed logit model of dative responses}

We used the procedure described in Experiment 1 to fit a mixed logit model to the within-language dative priming dataset. From the previous model we retained Prime Type as a predictor and added School to the random effects structure. The final model therefore comprised Prime Type (Baseline/Dative) as a fixed factor, random intercepts for item, target verb, and participant, as well as by-participant and by-school random effects of Prime Type. Again, we added meancentred English Score as a covariate, including its interaction with Prime Type. 
Table 7 Frequency and proportion of passive, active, and 'other' responses by prime condition, based on strict and lenient scoring schemes

\begin{tabular}{|c|c|c|c|c|c|c|}
\hline & \multicolumn{2}{|l|}{ Baseline } & \multicolumn{2}{|l|}{ Active } & \multicolumn{2}{|l|}{ Passive } \\
\hline & Frequency & Proportion & Frequency & Proportion & Frequency & Proportion \\
\hline \multicolumn{7}{|l|}{ Strict scoring } \\
\hline Passive response & 27 & 0.03 & 29 & 0.04 & 48 & 0.06 \\
\hline Active response & 655 & 0.80 & 663 & 0.81 & 634 & 0.77 \\
\hline Other response & 135 & 0.17 & 124 & 0.14 & 142 & 0.17 \\
\hline \multicolumn{7}{|l|}{ Lenient scoring } \\
\hline Passive response & 73 & 0.09 & 81 & 0.10 & 107 & 0.13 \\
\hline Active response & 655 & 0.80 & 663 & 0.81 & 634 & 0.77 \\
\hline Other response & 89 & 0.11 & 72 & 0.09 & 83 & 0.10 \\
\hline
\end{tabular}

Consistent with the observed $8 \%$ increase in the rate of $\mathrm{PO}$ production in the $\mathrm{PO}$ priming condition relative to baseline, Table 10 shows a positive coefficient for the predictor Prime Type: PO Dative. As zero falls outside the $95 \%$ confidence interval for this coefficient, we can conclude that there is evidence for a within-language $\mathrm{PO}$ priming effect. The model results reveal no main effect of English Score on PO production. Furthermore, there was no evidence for an interaction between English Score and Prime Type, suggesting that the within-L1 PO priming effect was independent of L1 proficiency.

\section{Exploratory combined analysis of Experiments 1 and 2}

To determine whether within-language structural priming was stronger than between-language priming for PO datives, we conducted an exploratory combined analysis of the binary response data from Experiment 1 and Experiment 2. We created a mixed logit model to predict the log-likelihood of producing a PO dative as a function of the fixed factors Prime
Type (Baseline/Dative), Prime Language (Irish/English), and, importantly, their interaction. The reference level for the Prime Language variable was Irish (i.e. the cross-linguistic priming condition, since the target language was always English). The model included separate random effects of Prime Type by school $(\mathrm{n}=3)$, and by participant $(\mathrm{n}=105)$, as well as random intercepts for target verb and item.

The negative intercept shown in Table 11 reflects a baseline preference for the DO dative in the Irish priming condition (i.e. Experiment 1). When we combine the datasets from both experiments, the model results indicate a main effect of Prime Type on the log-likelihood of a PO response, based on a $95 \%$ confidence interval. This is consistent with the numerical trend observed in both experiments, whereby more English PO datives were produced following an Irish dative or English PO prime, relative to the non-dative baseline. The interaction of interest, between Prime Type and Prime Language, has a positive coefficient but a $95 \%$ confidence interval that encompasses zero. Thus, we did not find evidence that

Table 8 Summary of fixed effects in the mixed logit model $(\mathrm{N}=2248$, log-likelihood $=-349.9)$

\begin{tabular}{lcccc}
\hline Predictor & Coefficient & SE & $z$ value & $95 \%$ CI \\
\hline Intercept & -3.99 & 0.40 & -9.87 & -4.79 to -3.20 \\
Prime type: passive & 0.15 & 0.59 & 0.25 & -1.01 to 1.30 \\
Prime type: active & -0.22 & 0.58 & -0.39 & -1.36 to 0.91 \\
English score & 0.50 & 0.45 & 1.10 & -0.39 to 1.39 \\
Prime type: passive $\times$ English score & 0.05 & 0.59 & 0.10 & -1.11 to 1.22 \\
Prime type: active $\times$ English score & 0.01 & 0.58 & 0.01 & -1.12 to 1.13 \\
\hline
\end{tabular}

The intercept represents the log-odds of a passive response in the baseline condition (NP $+\mathrm{NP})$ for a participant with an average English score

$S E$ standard error; $C I$ confidence interval 
Table 9 Frequency and proportion of prepositional-object (PO), double-object (DO), and 'other' responses by prime condition

\begin{tabular}{llllll}
\hline & \multicolumn{2}{l}{ Baseline } & & & Dative \\
\cline { 2 - 3 } \cline { 5 - 5 } & Frequency & Proportion & & Frequency & Proportion \\
\hline $\begin{array}{l}\text { response } \\
\text { DO } \\
\text { response }\end{array}$ & 355 & 0.30 & 443 & 0.38 \\
$\begin{array}{c}\text { Other } \\
\text { response }\end{array}$ & 431 & 0.37 & 403 & 0.34 \\
\hline
\end{tabular}

the priming effect was stronger in Experiment 2 than in Experiment 1 (Table 11).

\section{General discussion}

The present study investigated between- and withinlanguage structural priming of passives and datives, using written sentence generation in a classroom setting. In Experiment 1, bilingual Irish speakers read Irish primes and generated sentences in English to describe target pictures. Prime and target sentences were always semantically unrelated, with non-equivalent main verbs. In Experiment 2, an age-matched control group read the same prime sentences in English, and completed the same picture description task.

Based on previous studies supporting a lessrestricted account of shared syntax in bilinguals (e.g., Shin and Christianson 2009; Kutasi et al. 2018), we predicted that the Irish dative would prime production of the English PO dative in Experiment 1. The Irish dative shares some elements of constituent order with the English prepositional-object dative, although the two constructions differ with respect to surface constituent structure (NP NP vs. NP PP) and position of the main verb. Consistent with our hypothesis, Experiment 1 showed a small increase in the proportion of English PO datives produced after an Irish dative prime, relative to a non-dative baseline. This appeared to be driven by participants who rated their Irish proficiency the highest (averaged across modalities). Importantly, higher proficiency was not associated with a baseline preference for PO datives in English; on the contrary, more proficient participants showed a bias towards the DO (doubleobject) dative at baseline.

The interaction between cross-linguistic priming and proficiency in Experiment 1 is similar to a finding reported by Bernolet et al. (2013). They examined self-rated L2 proficiency as a predictor of structural priming magnitude in Dutch-English bilinguals, and found a robust positive correlation. Indeed, less proficient participants did not show any between-language priming for genitives in their study. Contrastingly, Kutasi et al. (2018) reported a main effect of Scottish Gaelic proficiency on passive production in English, but no interaction between proficiency and priming. However, as they noted in their discussion, a more heterogeneous sample might be required to investigate the effect of proficiency systematically.

Based on the results obtained with late bilinguals, Bernolet et al. (2013) posited that the interaction between L2 proficiency and cross-linguistic priming arises from the progressive abstraction of structures across languages. That is, L2 learners begin with language-specific, item-based representations for new syntactic structures, which gradually become integrated with existing representations of similar

Table 10 Summary of fixed effects in the mixed logit model $(\mathrm{N}=2152$, log-likelihood $=-955.9)$

\begin{tabular}{lcccc}
\hline Predictor & Coefficient & SE & $z$ value & 95\% CI \\
\hline Intercept & -1.74 & 0.83 & -2.10 & -3.37 to -0.11 \\
Prime type: PO dative & 0.58 & 0.16 & 3.53 & 0.26 to 0.90 \\
English score & -0.27 & 0.46 & -0.57 & -1.18 to 0.64 \\
Prime type: PO dative $\times$ English score & 0.30 & 0.19 & 1.58 & -0.07 to 0.69 \\
\hline
\end{tabular}

The intercept represents the log-odds of a PO dative response in the baseline condition $(\mathrm{NP}+\mathrm{NP}+\mathrm{NP})$ for a participant with an average English score

$S E$ standard error, $C I$ confidence interval 
Table 11 Summary of fixed effects in the mixed logit model for Experiments 1 and 2 combined ( $\mathrm{N}=4068$, loglikelihood $=-1785.8$ )

\begin{tabular}{lcccc}
\hline Predictor & Coefficient & SE & $z$ value & $95 \%$ CI \\
\hline Intercept & -2.28 & 0.77 & -2.94 & -3.80 to -0.76 \\
Prime type: dative & 0.32 & 0.15 & 2.06 & 0.02 to 0.62 \\
Prime language: English & 0.80 & 0.87 & 0.92 & -0.90 to 2.50 \\
Prime type: dative $\times$ prime language: English & 0.25 & 0.18 & 1.34 & -0.11 to 0.61 \\
\hline
\end{tabular}

The intercept represents the log-odds of a PO response in the baseline condition (NP $+\mathrm{NP})$ when the language of priming is Irish $S E$ standard error, $C I$ confidence interval

structures in L1. This account assumes that highly proficient bilinguals activate the same representations when encoding similar syntactic structures in their two languages, giving rise to a cross-linguistic structural priming effect. For less proficient bilinguals, betweenlanguage influences may be weaker or absent, because syntactic encoding involves separate representations for L1 and L2. The evidence we found for proficiencymodulated priming between Irish and English datives is compatible with this theoretical account, and supports the claim that even non-identical structures undergo a process of integration, given sufficient proficiency in both languages.

We contrast the results of Experiment 1 with those of Experiment 2, which suggested that within-language dative priming was unrelated to native language proficiency, as indexed by English exam scores. We acknowledge, however, that our non-equivalent proficiency measures limit the potential for direct comparison between experiments. Within a native language, the interaction between structural priming effects and linguistic proficiency might be a function of developmental stage. For example, Kidd (2012) found that grammatical knowledge and vocabulary predicted structural priming in 4-6 year-old native English speakers. By the time they reach adolescence, it is likely that native speakers have reached a plateau in syntactic acquisition, at least for the most common structures of their language, where abstract representations of those structures (e.g., the PO dative) are fully developed, facilitating structural priming between sentences with no lexical overlap. Our finding in Experiment 2, that within-L1 priming for $\mathrm{PO}$ datives was robust and independent of $\mathrm{L} 1$ proficiency, could thus reflect the efficiency of syntactic encoding for this structure, perhaps too close to ceiling to show an effect of proficiency. By this reasoning, priming for passives might be expected to show a comparatively larger effect of linguistic proficiency in adolescents, as the structure is less common and therefore should plateau later. While we did not find evidence in our data to support this, future work could address the issue using a priming paradigm that elicits more passives.

The developmental account of L2 syntactic acquisition (Hartsuiker and Bernolet 2017) has implications for the relative strength of between- versus withinlanguage priming effects, since it predicts that only highly proficient bilinguals with fully integrated structural representations should prime as strongly between languages as within. Several studies have found evidence for equivalent effects, independent of the priming language (e.g., Schoonbaert et al. 2007; Kantola and Van Gompel 2011). More recently, Hartsuiker et al. (2016) systematically investigated the issue in multilingual speakers and found that structural priming was always as strong between as within languages, supporting a fully shared syntax account (e.g., Hartsuiker et al. 2004). However, this finding did not hold for the less proficient bilinguals tested by Bernolet et al. (2013), leading them to conclude that the shared syntax model in fact represents the final state of bilingual memory, whereas the prevalence of language-specific representations in less proficient bilinguals results in weaker priming from L2 to L1 than within L2. Converging with Hartsuiker et al. (2016), we did not find evidence for a significant difference between Irish-to-English and within-English dative priming in terms of magnitude. This exploratory finding points to the engagement of shared, or tightly linked syntactic coding operations for Irish and English, at least in the more proficient bilinguals. 
In contrast to the results obtained for the dative construction, we did not find any passive priming. Kutasi et al. (2018) studied adolescent bilingual speakers of Scottish Gaelic and English and demonstrated cross-linguistic passive priming with one of the two Gaelic patient-focusing structures they tested. The present study tested a similar sample in terms of age and L2 proficiency, using materials adapted from the Scottish Gaelic study. As Scottish Gaelic shares many typological features with Irish, including VSO word order, we had expected to find a comparable effect of passive priming in Irish-speaking bilinguals in Experiment 1 . However, in our study the overall rate of full passives $(2.41 \%)$ was even lower than in the study by Kutasi and colleagues $(5.71 \%)$, and did not allow us to detect any existing priming effects. Further work is needed to provide a more conclusive test of Irish-toEnglish passive priming.

Although the overall rate of passive production in both of our experiments was very low, participants in Experiment 2 produced relatively more passive descriptions than participants in Experiment 1 $(4.23 \%)$. We speculate that this may reflect group differences in experience with written English, and by extension with the English passive, which is used more frequently in formal written text than in colloquial spoken language (Roland et al. 2007). Whilst adolescents educated through Irish undoubtedly gain exposure to written English outside of school, the distribution of passives in their input is unlikely to be equivalent to that of their English-educated peers, who consume a large volume of English educational texts, typically of a formal register. Notably, English exam results did not predict passive production in Experiment 2, indicating that the observed group difference in passive avoidance is unlikely to be related to general aptitude, but rather to experience. This explanation is compatible with the evidence that exposure to print, and specifically to the structural distributions of written language, influences syntactic choices in production (Montag and MacDonald 2015).

Despite the slightly higher rate of passive production overall in Experiment 2, we did not find evidence for a within-language passive priming effect. This is contrary to previous studies that have demonstrated structural priming of the passive in English, both in adults (e.g., Bock et al. 2007) and in children (e.g., Messenger et al. 2012). Our finding that the same participants exhibited priming for the PO dative in
Experiment 2 indicates that they were not resistant to structural priming per se. However, it may be that passive priming effects, which tend to be smaller in magnitude than dative priming effects (Mahowald et al. 2016), are more sensitive to variations in experimental design.

Although passive primes did not yield the expected priming effect in either study, we did obtain varying degrees of evidence for dative priming both within and between languages using a classroom-based, written sentence generation paradigm. This finding might encourage other researchers to adopt similar approaches, in order to assess theories of language processing in samples with different educational and language backgrounds to those typically tested in psycholinguistic studies. Classroom-based testing may require some adaptations of standard protocols, such as written rather than spoken response elicitation, and very carefully formulated instructions, while the increased likelihood of distraction remains difficult to avoid. Nevertheless, the present study may be taken to demonstrate the feasibility and potential of using classroom settings in priming research. Compared to web-based testing, which also allows for the efficient acquisition of large datasets, group testing offers the benefit of closer observation and tighter control of participants' behaviour during the experiment.

In conclusion, this study investigated cross-linguistic structural priming in bilingual adolescents receiving their education through Irish, which is typologically distant from English (the culturally dominant language in Ireland). We found that selfreported Irish proficiency predicted the strength of dative priming from Irish to English, in-line with previous evidence from Dutch-English bilinguals. A control experiment conducted at English-medium secondary schools showed a comparable within-L1 priming effect for PO datives. This was not modulated by L1 proficiency, possibly because the processing of relatively common structures in L1 was close to ceiling. Our findings are compatible with a developmental account of L2 syntactic acquisition, which assumes that the shared representations necessary for between-language priming emerge with increasing proficiency.

Acknowledgements Open access funding provided by Max Planck Society. We would like to thank the students who volunteered to participate in this study, and their teachers. We 
would also like to thank Beverley Hartigan and her colleagues for their Irish translations and language advice.

\section{Compliance with ethical standards}

Conflict of interest On behalf of all authors, the corresponding author states that there is no conflict of interest.

Open Access This article is distributed under the terms of the Creative Commons Attribution 4.0 International License (http:// creativecommons.org/licenses/by/4.0/), which permits unrestricted use, distribution, and reproduction in any medium, provided you give appropriate credit to the original author(s) and the source, provide a link to the Creative Commons license, and indicate if changes were made.

\section{Appendix 1}

See Fig. 6.

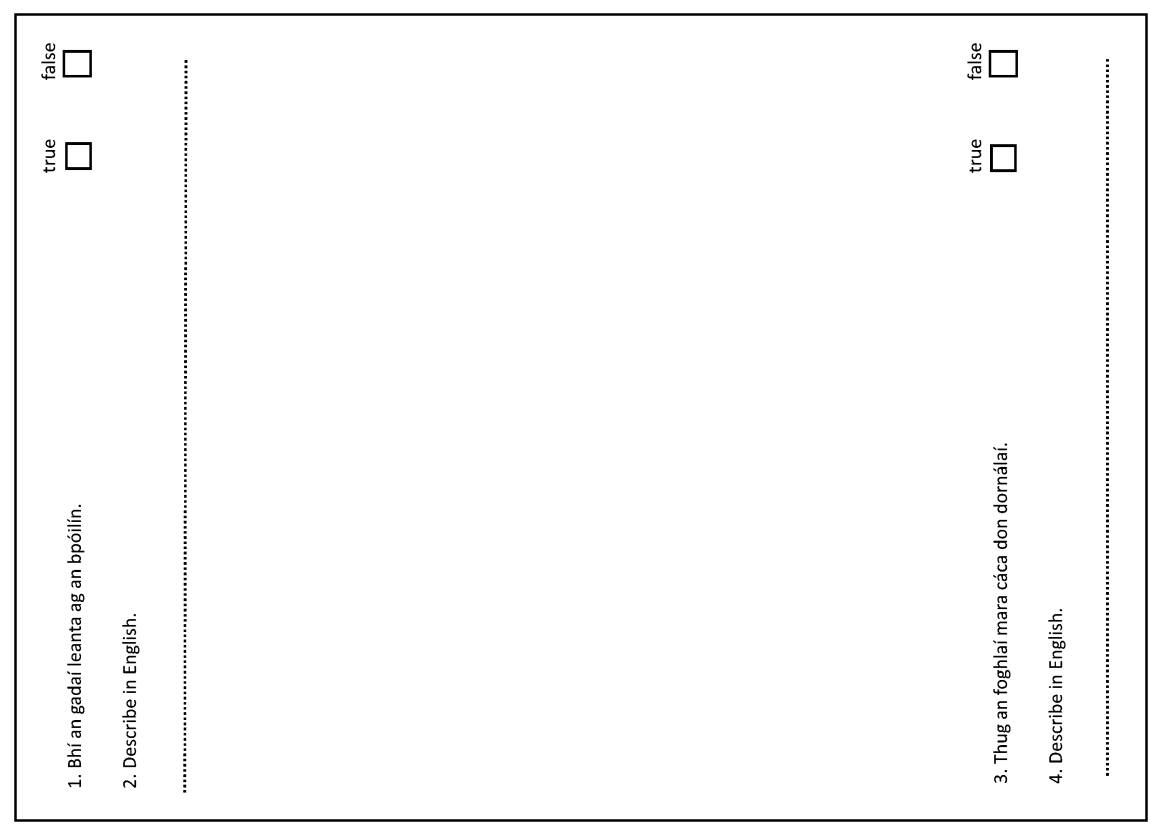

Fig. 6 Sample page from answer booklet 


\section{Appendix 2: Irish Language History Questionnaire}

Participant Number:

Date:

Name:

Date

of

Birth:

Gender:

1) Age when you...

Began Acquiring Irish:

Began Reading in Irish:

Began Writing in Irish:

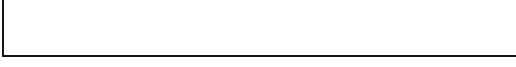

2) How often do you use Irish in conversation? Daily Weekly

(Please circle as appropriate) Monthly Rarely

3) Is Irish used in your home?

Yes

No

If yes, by who? (Please circle all that apply)

Parents/ Primary Caregiver Grandparents Other (Please Specify):

Siblings Child-minder

4) How often do you engage with Irish Media (e.g., TV, Radio, or Newspapers)?

Daily Weekly Monthly Rarely

5) Please rate the level of your Irish proficiency for each section: $1=$ Very Low and $7=$ Very Comfortable

\section{Very Poor}

\section{Very Comfortable}

$\begin{array}{llllllll}\text { Reading: } & 1 & 2 & 3 & 4 & 5 & 6 & 7 \\ \text { Speaking: } & 1 & 2 & 3 & 4 & 5 & 6 & 7 \\ \text { Writing: } & 1 & 2 & 3 & 4 & 5 & 6 & 7\end{array}$

Understanding

Spoken Language: $\quad \begin{array}{llllllll}1 & 2 & 3 & 4 & 5 & 6 & 7\end{array}$

6) Excluding Irish and English, can you hold a conversation in any other languages? Yes / No
If
yes,
please
specify
which
language(s):

7) Which grade did you receive for Irish in the Junior Cert? Grade: Level:

Which grade did you receive for English in the Junior Cert? Grade: Level: 


\section{References}

Bates, D., Maechler, M., Bolker, B., \& Walker, S. (2013). lme4: Linear mixed-effects models using Eigen and S4. R package version, 1(4).

Bernolet, S., Collina, S., \& Hartsuiker, R. J. (2016). The persistence of syntactic priming revisited. Journal of Memory and Language, 91, 99-116.

Bernolet, S., Hartsuiker, R. J., \& Pickering, M. J. (2007). Shared syntactic representations in bilinguals: Evidence for the role of word-order repetition. Journal of Experimental Psychology. Learning, Memory, and Cognition, 33(5), 931.

Bernolet, S., Hartsuiker, R. J., \& Pickering, M. J. (2013). From language-specific to shared syntactic representations: The influence of second language proficiency on syntactic sharing in bilinguals. Cognition, 127(3), 287-306.

Bock, J. K. (1986). Syntactic persistence in language production. Cognitive Psychology, 18(3), 355-387.

Bock, K. (1989). Closed-class immanence in sentence production. Cognition, 31(2), 163-186.

Bock, K., Dell, G. S., Chang, F., \& Onishi, K. H. (2007). Persistent structural priming from language comprehension to language production. Cognition, 104(3), 437-458.

Bock, K., \& Loebell, H. (1990). Framing sentences. Cognition, 35(1), 1-39.

Branigan, H. P., \& McLean, J. F. (2016). What children learn from adults' utterances: An ephemeral lexical boost and persistent syntactic priming in adult-child dialogue. Journal of Memory and Language, 91, 141-157.

Branigan, H. P., \& Pickering, M. J. (2017). An experimental approach to linguistic representation. Behavioral and Brain Sciences, 40.

Branigan, H. P., Pickering, M. J., \& Cleland, A. A. (1999). Syntactic priming in written production: Evidence for rapid decay. Psychonomic Bulletin and Review, 6(4), 635-640.

Eurobarometer 386, S. (2012). Europeans and their Languages. European Commission.

Gries, S. T., \& Stefanowitsch, A. (2004). Extending collostructional analysis: A corpus-based perspective on alternations'. International Journal of Corpus Linguistics, 9(1), 97-129.

Hartsuiker, R. J., Beerts, S., Loncke, M., Desmet, T., \& Bernolet, S. (2016). Cross-linguistic structural priming in multilinguals: Further evidence for shared syntax. Journal of Memory and Language, 90, 14-30.

Hartsuiker, R. J., \& Bernolet, S. (2017). The development of shared syntax in second language learning. Bilingualism: Language and Cognition, 20(2), 219-234.

Hartsuiker, R. J., Pickering, M. J., \& Veltkamp, E. (2004). Is syntax separate or shared between languages? Cross-linguistic syntactic priming in Spanish-English bilinguals. Psychological Science, 15(6), 409-414.

Kantola, L., \& van Gompel, R. P. (2011). Between-and withinlanguage priming is the same: Evidence for shared bilingual syntactic representations. Memory and Cognition, 39(2), 276-290.
Kidd, E. (2012). Individual differences in syntactic priming in language acquisition. Applied Psycholinguistics, 33(2), 393-418.

Kutasi, T., Suffill, E., Gibb, C.L., Sorace, A., Pickering, M.J., \& Branigan, H.P., (2018). Shared representation of passives across Scottish Gaelic and English: Evidence from structural priming. Journal of Cultural Cognitive Science.

Lapata, M. (1999). Acquiring lexical generalizations from corpora: a case study for diathesis alternations. In Proceedings of the 37th annual meeting of the Association for Computational Linguistics on Computational Linguistics (ACL '99) (pp. 397-404). Association for Computational Linguistics, Stroudsburg, PA, USA.

Lemhöfer, K., \& Broersma, M. (2012). Introducing LexTALE: A quick and valid lexical test for advanced learners of English. Behavior Research Methods, 44(2), 325-343.

Loebell, H., \& Bock, K. (2003). Structural priming across languages. Linguistics, 41(5; ISSU 387), 791-824.

Mahowald, K., James, A., Futrell, R., \& Gibson, E. (2016). A meta-analysis of syntactic priming in language production. Journal of Memory and Language, 91, 5-27.

Messenger, K., Branigan, H. P., McLean, J. F., \& Sorace, A. (2012). Is young children's passive syntax semantically constrained? Evidence from syntactic priming. Journal of Memory and Language, 66(4), 568-587.

Montag, J. L., \& MacDonald, M. C. (2015). Text exposure predicts spoken production of complex sentences in 8-and 12-year-old children and adults. Journal of Experimental Psychology: General, 144(2), 447.

Pandharipande, R. (2002). Minority matters: issues in minority languages in India. International Journal on Multicultural Societies, 4(2), 213-234.

Roland, D., Dick, F., \& Elman, J. L. (2007). Frequency of basic English grammatical structures: A corpus analysis. Journal of Memory and Language, 57(3), 348-379.

Schoonbaert, S., Hartsuiker, R. J., \& Pickering, M. J. (2007). The representation of lexical and syntactic information in bilinguals: Evidence from syntactic priming. Journal of Memory and Language, 56(2), 153-171.

Shin, J. A., \& Christianson, K. (2009). Syntactic processing in Korean-English bilingual production: Evidence from cross-linguistic structural priming. Cognition, 112(1), 175-180.

Slatinská, A. (2017). The Irish language: A unique part of Irish life and cultural revitalisation and protection. Intercultural Relations, 2, 2.

Van Gompel, R. P., \& Arai, M. (2018). Structural priming in bilinguals. Bilingualism: Language and Cognition, 21(3), 448-455.

Publisher's Note Springer Nature remains neutral with regard to jurisdictional claims in published maps and institutional affiliations. 